2 Barbato A, Frischer T, Kuehni CE, et al. Primary ciliary dyskinesia: a consensus statement on diagnostic and treatment approaches in children. Eur Respir J 2009; 34: 1264-1276.

3 Bush A, Chodhari R, Collins N, et al. Primary ciliary dyskinesia: current state of the art. Arch Dis Child 2007; 92: 1136-1140.

4 Lucas JS, Walker WT, Kuehni CE, et al. Primary ciliary dyskinesia. In: Cordier J-F, ed. Orphan Lung Diseases. Eur Respir Monogr 2011; 54: 201-217.

5 Jorissen M, Willems T, Van der Schueren B, et al. Ultrastructural expression of primary ciliary dyskinesia after ciliogenesis in culture. Acta Otorhinolaryngol Belg 2000; 54: 343-356.

6 Shoemark A, Dixon M, Corrin B, et al. Twenty-year review of quantitative transmission electron microscopy for the diagnosis of primary ciliary dyskinesia. J Clin Pathol 2012; 65: 267-271.

7 Hirst RA, Rutman A, Williams G, et al. Ciliated air-liquid cultures as an aid to diagnostic testing of primary ciliary dyskinesia. Chest 2010; 138: 1441-1447.

8 Jorissen M, Willems T, Van der Schueren B. Ciliary function analysis for the diagnosis of primary ciliary dyskinesia: advantages of ciliogenesis in culture. Acta Otolaryngol 2000; 120: 291-295.

9 Omran H, Loges NT. Immunofluorescence staining of ciliated respiratory epithelial cells. Methods Cell Biol 2009; 91: 123-133.

10 Marthin JK, Mortensen J, Pressler T, et al. Pulmonary radioaerosol mucociliary clearance in diagnosis of primary ciliary dyskinesia. Chest 2007; 132: 966-976.

11 Bennett WD, Laube BL, Corcoran T, et al. Multisite comparison of mucociliary and cough clearance measures using standardized methods. J Aerosol Med Pulm Drug Deliv 2013; 26: 157-164.

12 Fleming JS, Conway JH, Majoral C, et al. The use of combined single photon emission computed tomography and X-ray computed tomography to assess the fate of inhaled aerosol. J Aerosol Med Pulm Drug Del 2011; 24: 49-60.

\title{
Severe influenza A H7N9 pneumonia with rapid virological response to intravenous zanamivir
}

\author{
To the Editor:
}

In March 2013, a novel influenza A H7N9 virus of avian origin was reported to cause severe pneumonia in mainland China [1-3]. While the great majority of patients were treated with oral oseltamivir, impaired gastrointestinal absorption in critically ill patients, the lack of virological response among patients requiring extracorporeal membrane oxygenation (ECMO) and detection of an amino acid change (arginine to lysine at residue 292 in N2) in the viral neuraminidase associated with drug resistance in two H7N9 strains are major concerns $[1,4]$. Here, we report a case of severe pneumonia due to avian influenza A H7N9 requiring ECMO support; there was rapid clinical and virological response following intravenous zanamivir therapy.

On November 21, 2013, in Hong Kong, China, a 36-year-old Indonesian female began to have fever, malaise and cough. She had been previously healthy except for a history of vitiligo. She sought medical advice from two clinics and was given symptomatic treatment. She attended the accident and emergency department of a regional hospital in Hong Kong on November 27 when the symptoms deteriorated. Her temperature was $40.0^{\circ} \mathrm{C}$, blood pressure was $120 / 63 \mathrm{mmHg}$, pulse was 116 beats $\cdot \mathrm{min}^{-1}$, respiratory rate was 18 breaths $\cdot \min ^{-1}$ and oxygenation was $97 \%$ while breathing ambient air. A chest radiograph showed right lower zone consolidation.

After admission, there was persistent fever, rapid progression of the radiological infiltrates to both lungs and development of parapneumonic effusion despite treatment with intravenous amoxicillin-clavulanate, ceftriaxone, azithromycin and oral oseltamivir (75 mg twice daily, total of two doses given). Endotracheal intubation and mechanical ventilation were required on November 29. Desaturation worsened and she was put on ECMO on November 30. Initial microbiological workup including sputum culture, blood culture, urine for Legionella and pneumococcal antigens, two nasopharyngeal swabs for influenza A and B antigens, and tracheal aspirate for human metapneumovirus, Mycoplasma pneumoniae, Chlamydophila pneumoniae and Mycobacterium tuberculosis by PCR were negative.

In view of the rapid disease progression, the respiratory specimens were subjected to testing for avian influenza viruses [5]. PCR identified presence of H7N9 in all the respiratory specimens (throat swab, nasopharyngeal swabs and aspirates, tracheal aspirate, and pleural fluid) collected since hospitalisation. No H7N9 was detected in urine or rectal swabs. PCR for influenza A subtypes H1, H3 and H5 of the nasopharyngeal swabs and tracheal aspirates was negative. Therapy with intravenous zanamivir $600 \mathrm{mg}$ 


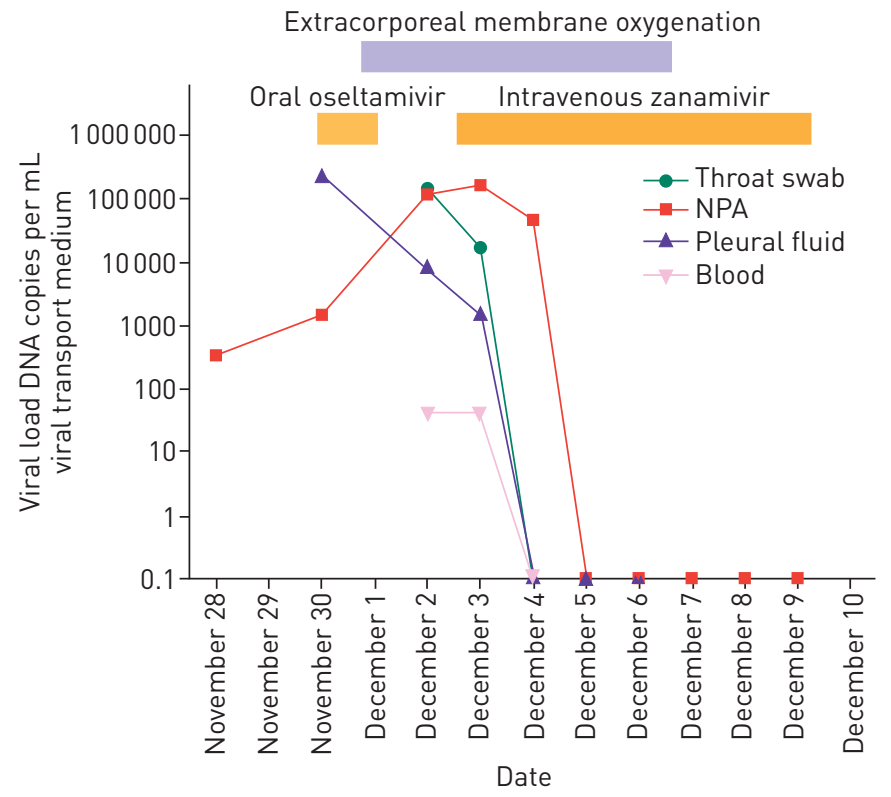

FIGURE 1 Temporal changes in H7N9 viral load after intravenous zanamivir therapy. NPA: nasopharyngeal aspirate.

every $12 \mathrm{~h}$ was started on December 2. Quantitative PCR targeting the matrix gene demonstrated that the viral loads had declined rapidly following zanamivir therapy (fig. 1). Sequence analysis of the neuraminidase gene of the virus recovered from tracheal aspirate showed no resistance-associated mutations. The patient's condition improved clinically; she was extubated after 2 days and ECMO was discontinued on December 6. Subsequently, it was revealed that the patient had a history of buying, slaughtering and eating a chicken in Shenzhen, China, on November 17.

Here, we document, for the first time, rapid virological clearance in a patient with severe H7N9 pneumonia requiring ECMO. In the absence of comparative clinical studies, the optimal antiviral therapy for pneumonia caused by H7N9 remains uncertain. Genetic analysis and in vitro susceptibility testing demonstrated that most strains of H7N9 are susceptible to neuraminidase inhibitors oseltamivir and zanamivir, but resistant to adamantanes [1]. In patients with respiratory failure, inhaled neuraminidase inhibitors are unlikely to be helpful because the drugs are not expected to reach the consolidated lungs. For hospitalised patients with suspected or confirmed H7N9 influenza, both oral oseltamivir and intravenous zanamivir are currently considered to be acceptable alternatives for therapy [6, 7]. Although enterically administered oseltamivir is well absorbed in critically ill influenza patients, intravenous zanamivir is preferred for patients with gastric stasis, malabsorption or gastrointestinal bleeding [6]. Antiviral therapy in the present patient was switched to intravenous zanamivir because a study by $\mathrm{Hu}$ et al. [4] found that patients on ECMO responded poorly to enterically administered oseltamivir and that neuraminidaseresistant mutants may emerge during oseltamivir therapy under such clinical circumstances. In accordance with previous observations, decline in viral loads in our patient correlated with clinical improvement $[1,4]$. Our findings are useful for informing the choice of antiviral therapy in future cases but further clinical trials would be required to confirm the observation.

0

@ERSpublications

Intravenous zanamivir may be the best treatment for influenza A H7N9 pneumonia in patients undergoing ECMO http://ow.ly/uIjN7

Pak-Leung $\mathrm{Ho}^{1}$, Wai-Ching $\mathrm{Sin}^{2}$, Jasper Fuk-Woo $\mathrm{Chan}^{1}$, Vincent Chi-Chung Cheng ${ }^{1}$ and Kwok-Hung Chan ${ }^{1}$ ${ }^{1}$ Carol Yu Center for Infection and Dept of Microbiology, University of Hong Kong, Hong Kong, China. ${ }^{2}$ Adult Intensive Care Unit, Queen Mary Hospital, Hong Kong, China.

Correspondence: Pak-Leung Ho, Division of Infectious Diseases, Dept of Microbiology and Carol Yu Centre for Infection, The University of Hong Kong, Queen Mary Hospital, Pokfulam, Hong Kong SAR, China.

E-mail: plho@hkucc.hku.hk

Received: Jan 092014 | Accepted after revision: March 032014 | First published online: April 22014 
Support statement: The work is supported by a commission grant from the Research Fund for the Control of Infectious Diseases of the Food and Health Bureau of the Hong Kong SAR Government.

Conflict of interest: Disclosures can be found alongside the online version of this article at erj.ersjournals.com

Acknowledgements: We thank the patient for giving written consent for publication.

References

1 To KK, Chan JF, Chen H, et al. The emergence of influenza A H7N9 in human beings 16 years after influenza A H5N1: a tale of two cities. Lancet Infect Dis 2013; 13: 809-821.

2 Chen Y, Liang W, Yang S, et al. Human infections with the emerging avian influenza A H7N9 virus from wet market poultry: clinical analysis and characterisation of viral genome. Lancet 2013; 381: 1916-1925.

3 Chan JF, To KK, Tse H, et al. Interspecies transmission and emergence of novel viruses: lessons from bats and birds. Trends Microbiol 2013; 21: 544-555.

$4 \mathrm{Hu}$ Y, Lu S, Song Z, et al. Association between adverse clinical outcome in human disease caused by novel influenza A H7N9 virus and sustained viral shedding and emergence of antiviral resistance. Lancet 2013; 381: $2273-2279$.

5 Wang W, Ren P, Mardi S, et al. Design of multiplexed detection assays for identification of avian influenza a virus subtypes pathogenic to humans by SmartCycler real-time reverse transcription-PCR. J Clin Microbiol 2009; 47: 86-92.

6 Centers for Disease Control and Prevention. Interim guidance on the use of antiviral agents for treatment of human infections with avian influenza A (H7N9) virus. www.cdc.gov/flu/avianflu/h7n9-antiviral-treatment.htm Date last accessed: January 9, 2014. Date last updated: September 30, 2013.

7 World Health Organization. Avian influenza A(H7N9) virus. www.who.int/influenza/human_animal_interface/ influenza_h7n9/en/ Date last accessed: January 9, 2014. Date last updated: May 31, 2013.

\section{Tuberculosis diagnostics: which target product profiles should be prioritised?}

\section{To the Editor:}

Globally, a third of all tuberculosis (TB) cases are not notified and many patients do not receive drug susceptibility testing (DST) [1]. New diagnostics can contribute to increased case detection, shorter diagnostic delay and reduced TB transmission. While the Xpert MTB/RIF assay (Cepheid Inc., Sunnyvale, CA, USA) is a much needed breakthrough [2], it may not reach lower tiers of the healthcare system [3] and doesn't meet all needs (e.g. cannot detect resistance against multiple drugs).

Several promising diagnostics are under development and companies are showing interest in TB products, inspired by the success of Xpert MTB/RIF [4]. But which new TB diagnostics should they invest in, and what is the potential market size for these products? Stakeholders have expressed a need for different products, including a test for childhood TB [5], a simple point-of-care-test for active pulmonary TB [6], a molecular smear replacement test [7], DST for expected new drug regimens [8], predictive biomarkers for latent TB infection (LTBI) [9], and treatment monitoring [10].

Given the variety of these needs, it is important for product developers to have access to: 1) a clearly identified list of diagnostics that are considered high priority by the TB community; 2) well developed, detailed target product profiles (TPPs) for priority diagnostics; and 3) up-to-date market size estimates for the priority TPPs. These issues are being addressed by ongoing activities, supported by the Bill and Melinda Gates Foundation (Seattle, WA, USA) and other partners [3, 7, 11].

TPPs are useful to align the end-users needs with the targets and specifications that product developers should meet. TPPs should state the clinical purpose of a test, goal to be met (e.g. start treatment), target population, implementation level in the healthcare system, and likely end-users (unpublished observations).

In 2013, participants at a TB Modelling and Analysis Consortium (TB MAC) meeting on diagnostics identified a list of diagnostic needs that could be developed into TPPs (unpublished observations). Using this list of nine potential TPPs (fig. 1), we conducted a priority-setting exercise to identify the highest priority tests for TPP development and investment in research and development. For each of the potential TPPs, hereafter called TPPs, 10 criteria were used to set priorities, including prioritisation by key stakeholders, potential impact of the test on TB transmission, morbidity and mortality, market potential and implementation and scalability of the test. 